\title{
Die Haut als Spiegel des Stoffwechsels
}

\author{
Hautprobleme im Zusammenhang mit Diabetes sind keine Seltenheit und kommen schon \\ bei Kindern vor. Für einige dieser dermatologischen Krankheitsbilder ist der Diabe- \\ tes kausal, andere sind nur mit dem Diabetes assoziiert. Auch eine Insulinpumpe
} kann kutane Komplikationen verursachen.

In Deutschland leiden ca. 30.000 Kinder und Jugendliche an einem Typ-1-Diabetes. „Man schätzt, dass ähnlich wie bei erwachsenen Typ-2-Diabetikern zwei Drittel dieser jungen Patienten an mindestens einer Hauterkrankung leiden“, so Privatdozent Hagen Ott, Leiter der Dermatologischen Abteilung am Kinderkrankenhaus Auf der Bult in Hannover. Das Spektrum der dermatologischen Krankheitsbilder ist breit. Grundsätzlich muss man zwischen Indikator-Dermatosen, die mit der Stoffwechselerkrankung in einem kausalen Zusammenhang stehen, pumpenbedingten Dermatosen („Pumps bumps“) und mit dem Diabetes assoziierten Hauterkrankungen unterscheiden.

\section{Necrobiosis lipoidica vor allem prätibial}

Ein für den Diabetes typisches dermatologisches Krankheitsbild ist die Necrobiosis lipoidica. Dabei handelt es sich um eine chronische, nekrotisierende, granulomatöse Hauterkrankung, die bei Typ-1-Diabetikern und bei Frauen sehr viel häufiger auftritt als bei Typ-2-Diabetikern bzw. Männern. Die Erkrankung kann vor, mit oder auch erst nach der Diagnosestellung „Diabetes“ manifest werden. Typische Lokalisation sind die Streckseiten der Unterschenkel, seltener sind Gesicht, Stamm oder Arme betroffen. Histologisch finden sich Granulome mit zentraler Nekrobiose. Die Erkrankung bedeutet für die Betroffenen eine kosmetische Beeinträchtigung, ist aber meist nicht schmerzhaft. Sie kann aber chronisch verlaufen. „Vermeiden von Traumata und Wundmanagement sind die Basistherapie“, so Ott. Ansonsten sind topische Glukokortikoide oder topische Immunmodulatoren wie Tacrolimus wirksam.

\section{Granuloma anulare meist selbstlimitierend}

Ein ähnliches Krankheitsbild ist das Granuloma anulare, das aber bei Typ-2-Diabetikern häufiger ist als bei Typ-1-Diabetikern. Auch hierbei handelt es sich um eine nekrobiotische Entzündung unklarer Genese. Betroffen sind 0,3\% aller Diabetiker. Es tritt häufig nach einem Bagatelltrauma auf. Bei Diabetikern überwiegt die disseminierte Form mit heteroformer Morphologie. Auch hier stehen für die Therapie topische Steroide oder Immunmodulatoren zur Verfügung. „Gerade bei Kindern sollte man die besorgten Eltern aber darauf hinweisen, dass es sich um eine selbstlimitierende gutartige Erkrankung handelt, die fast immer ohne Narbenbildung abheilt, aber es gibt protrahierte, über Jahre andauernde Krankheitsverläufe“, so Ott.

\section{Acanthosis nigricans benigna}

Bei der Acanthosis nigricans benigna handelt es sich um eine symptomlose, großflächige „Wucherung“ der Haut, die als kutanes Zeichen einer Insulinresistenz angesehen werden kann ( s. Abb. 1). Ganz selten ist diese Hautveränderung Ausdruck

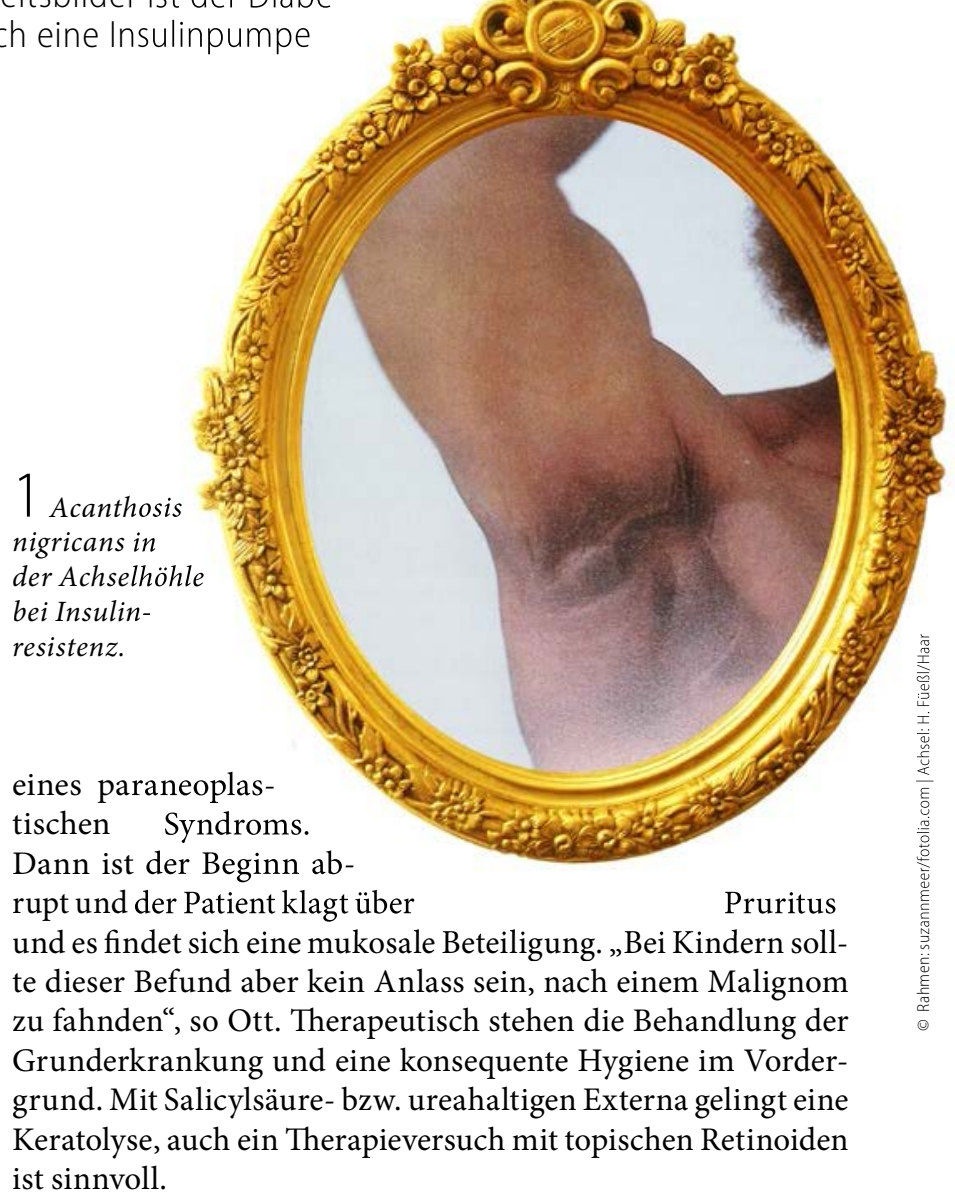

\section{Kutane Komplikationen bei Pumpen sind selten}

Bei Patienten mit einer Insulinpumpe sind kutane Komplikationen heute sehr selten. Am häufigsten werden Erytheme und Knoten als Ausdruck einer Lipohypertrophie beobachtet, Infektionen bzw. Abszesse sind eine Rarität. Bei Risikopatienten mit einem atopischen Ekzem muss vermehrt mit einem Kontaktekzem gerechnet werden, was eine topische Therapie mit einem Steroid oder Immunmodulator erfordert.

\section{Vitiligo ist diabetesassoziiert}

Die Vitiligo - die häufigste Depigmentierungserkrankung - ist nicht kausal mit dem Diabetes verknüpft, sondern als Autoimmunerkrankung nur diabetesassoziiert, wie auch andere Autoimmunerkrankungen. Gleiches gilt für die Psoriasis. Sie tritt bei Männern und Frauen gleich häufig auf. Wie bei anderen $\mathrm{Au}$ toimmunerkrankungen erfordert die Manifestation eine genetische Prädisposition. Eine topische Therapie mit einem Immunmodulator ist wirksam, aber Off-label-use.

Dr. med. Peter Stiefelhagen

Quelle: 51. DDG-Jahrestagung, 05.05.2016 in Berlin 